\title{
Preoperative anemia as a prognostic factor in patients with lung cancer: a systematic review and meta-analysis of epidemiological studies
}

\author{
Yang Liu, Yun-Peng Bai, Zi-Fang Zhou, Chang-Rui Jiang, Zhe Xu, Xiao-Xi Fan ${ }^{\bowtie}$ \\ Department of Thoracic Surgery, the First Affiliated Hospital of China Medical University, Shenyang, China. \\ $\triangle$ Corresponding author: Xiao-Xi Fan, M.D. Department of Thoracic Surgery, The First Affiliated Hospital of China Medical University Address: No. 155, \\ Nanjing Bei Street, Shenyang, Liaoning 110001, P. R. China. Phone: +86-024-83283170 E-mail: fanxx@cmu1h.com \\ (C) Ivyspring International Publisher. This is an open access article distributed under the terms of the Creative Commons Attribution (CC BY-NC) license \\ (https://creativecommons.org/licenses/by-nc/4.0/). See http://ivyspring.com/terms for full terms and conditions.
}

Received: 2018.08.22; Accepted: 2019.04.23; Published: 2019.05.12

\begin{abstract}
The evidence of current epidemiological studies investigating the relationship between preoperative anemia and progression of lung cancer (LC) patients remains controversial. The PubMed, EMBASE, and Web of Science databases were comprehensively searched by two independent authors to identify related epidemiological studies from inception through January 31, 2019. Similarly, two researchers separately extracted data and any differences were resolved by discussion. Summarized hazard ratios (HRs) and $95 \%$ confidence intervals $(\mathrm{Cls})$ were summarized with inverse variance weighted random effects meta-analysis. Heterogeneity among studies was assessed with the $I^{2}$ statistic. Twenty-two studies were included in this meta-analysis. As compared with LC patients without anemia, those with pre-operative anemia were at a 1.6-fold greater risk of death (summarized $\mathrm{HR}=1.58 ; 95 \% \mathrm{Cl}=1.44-1.75)$, with moderate heterogeneity $\left(I^{2}=53.1 \%\right.$ ). Funnel plot and statistical analyses showed no evidence of publication bias. Associations between pre-operative anemia and OS were broadly consistent across numerous subgroups analyses stratified by the study design, geographic location, number of cases, tumor, node, and metastasis (TNM) stage, histology, quality, and adjustment for potential confounders (age, sex, body mass index, TNM stage, histology, performance status, surgery, blood transfusion, and systemic inflammatory response markers). Similar patterns were observed in the sensitivity analyses. The results of meta-regression analysis suggested no evidence of significant heterogeneity between subgroups. In conclusion, pre-operative anemia was associated with poorer overall survival among LC patients.
\end{abstract}

Key words: pre-operative anemia, overall survival, lung cancer (LC) patients, meta-analysis

\section{Introduction}

Lung cancer (LC) is the leading cause of cancer-related death worldwide, accounting for approximately 1.59 million deaths in 2012 [1]. Non-small cell lung cancer (NSCLC) accounts for approximately $85 \%$ of all LCs and almost $70 \%$ of patients present with locally advanced or metastatic disease at the time of diagnosis [2, 3]. Despite advances in treatment, the 5-year overall survival (OS) rate remains at less than 20\% [4]. Established predictors of OS among LC patients include age at diagnosis, sex, tumor stage, histologic type, and certain genetic mutations [4]. However, monitoring of these factors is either invasive or costly and provides insufficient evidence for validation [5-7]. Therefore, economical and convenient clinical biomarkers for individualized prognosis and prediction of treatment outcomes are still urgently required for LC patients.

Hemoglobin $(\mathrm{Hb})$ is a biochemical biomarker commonly assessed during clinical examinations [8]. Notably, anemia and low $\mathrm{Hb}$ levels are quite common in patients with malignant tumors, including LC, and might be multifactorial. Indeed, low $\mathrm{Hb}$ levels, 
particularly in patients with more aggressive tumors, may also be related to complex interactions among the immune system, tumor microenvironment, and cancer cells $[9,10]$. Although the number of studies exploring the prognostic role of preoperative anemia and $\mathrm{Hb}$ levels has been increasing, the results are inconsistent and often based on small samples. Of note, Caro et al. [11] summarized the evidence of this topic in 2001 and several later reports have indicated that preoperative anemia or low $\mathrm{Hb}$ levels are associated with poor survival among LC patients [8, 12-33]; however, contradictory or null reports also exist [34-37].

Importantly, to the best of our knowledge, the prognostic value of preoperative anemia in LC patients has not been investigated in any systematic review or meta-analysis since 2001. Therefore, the purpose of this study was to summarize and update the currently available evidence from epidemiological studies regarding the association between pre-operative anemia and OS of LC patients.

\section{Material and Methods}

\section{Search strategy}

Two authors (YL and X-XF) independently and systematically searched the PubMed (https://www.ncbi.nlm.nih.gov/pubmed/), Embase (https://www.elsevier.com/solutions/embase-biom edical-research), and Web of Science (https://www.webofknowledge.com) databases from inception to the end of January 2019 for relevant epidemiological studies investigating the association between pre-operative anemia and progression of LC using the following search algorithm: "(anemia OR hemoglobin OR hematocrit OR transfusion OR blood cell OR hematology) AND (lung OR pulmonary) AND (cancer OR neoplasm OR carcinoma OR tumor)". A manual review of references from eligible systematic and narrative reviews was also performed. A meta-analysis was planned, conducted, and reported according to the guidelines of the Meta-Analysis of Observational Studies in Epidemiology group [38].

\section{Study selection and exclusion}

The following inclusion criteria were used: (i) observational or experimental study design; (ii) studies that evaluated the association between preoperative anemia status or $\mathrm{Hb}$ level and prognostic outcomes of LC patients; (iii) reported at least one of the outcomes of interest (i.e., OS, cancer-specific mortality, disease-free survival, event-free survival, progression-free survival, and recurrence-free survival); and (iv) studies that included the hazard ratio (HR) or relative risk (RR) with a 95\% confidence intervals $(\mathrm{CI})$, or reported sufficient data to calculate those risk estimates. The following exclusion criteria were used: (i) reviews without original data, ecological studies, editorials, and case reports; and (ii) studies that reported risk estimates without a 95\% CI (e.g., could not be included in the statistical summary). If multiple studies had a duplicate patient cohort, only that with the largest sample size was included for the same outcome. If multiple studies had a duplicate patient cohort as well as the same sample size, only that with the longest follow-up duration was included for the same outcome. However, multiple studies with duplicate patient cohorts but with different outcomes of interest were included for analysis separately for each outcome.

The titles and abstracts of the retrieved articles were checked for relevancy before the full-text article was examined. The relevant data were extracted from the complete articles. Also, the bibliographies of the selected articles were manually reviewed. The titles, abstracts, and full texts of the resulting articles were examined in detail by two independent authors ( $\mathrm{YL}$ and $\mathrm{X}-\mathrm{XF}$ ) and discrepancies were resolved by consensus.

\section{Data abstraction and quality assessment}

The following information was extracted from each included study by a single investigator $(\mathrm{YL})$ : first author, publication year, country, study design, number of patients, outcomes characteristics, and study-specific adjusted risk estimates with 95\% CIs. For risk estimates, if both univariate and multivariate analyses were provided, data from multivariate analysis were extracted; otherwise, data from univariate analysis were used. The predefined primary outcome was progression-free survival and the secondary outcome was OS. Extracted data were entered into a standardized Excel (Microsoft Corporation, Redmond, WA, USA) file. Subsequently, an independent author (Y-PB) checked the data and all differences were resolved by a third investigator $(\mathrm{X}-\mathrm{XF})$. Although five included studies were randomized controlled trials [22, 23, 33, 34, 37], each retrospectively analyzed the prognostic role of pre-operative anemia in the include patients. Therefore, two independent authors ( $\mathrm{YL}$ and $\mathrm{X}-\mathrm{XF}$ ) assessed the methodological quality of the included studies according to the Newcastle-Ottawa Scale [39].

\section{Statistical analysis}

The HR and 95\% CI are presented as summaries of the risk estimates of each study as calculated with a random effects model to investigate the association between preoperative anemia status and $\mathrm{Hb}$ levels 
with the progression of LC. For studies [12-14, 18, 22, 25-27] that set patients with anemia as the reference group, the counting method proposed by Hamling et al. [40] was used to recalculate the HR and 95\% CI. Heterogeneity across the studies was quantified using the $I^{2}$ statistic, which indicates significant heterogeneity when $I^{2}>50 \%$ [41]. Also, post hoc subgroup analyses was conducted according to the study design (retrospective vs. prospective), geographic location (Asia, Europe, and North America), tumor, node, and metastasis (TNM) stage (all, advanced stage, and early stage), histology (non-small cell vs. small cell), study quality (low vs. high risk), the median number of LC cases $(\geq 300$ vs. $<$ 300 ), and adjustments made for potential confounders (including age at surgery/diagnosis, sex, body mass index, TNM stage, histology, performance status, surgery, blood transfusion, and systemic inflammatory response markers). Heterogeneity between subgroups was evaluated by meta-regression analysis. Small study biases (e.g., publication bias) were assessed by visually inspecting a funnel plot and conducting tests according to Begg et al. [42] and Egger et al. [43] Sensitivity analyses were conducted by removing one study at a time to examine the effect of data from each study on the overall estimate. The sequential exclusion strategy proposed by Patsopoulos et al. [44] was used to determine whether the overall estimates were influenced by the substantial heterogeneity observed. Studies that accounted for the largest share of heterogeneity were sequentially and cumulatively excluded until $I^{2}$ was < $50 \%$. Then, further examinations were conducted to determine whether the risk estimates were consistent $[45,46]$. All statistical analyses were performed using the Stata statistical software package (ver. 12.0; StataCorp LP, College Station, TX, USA).

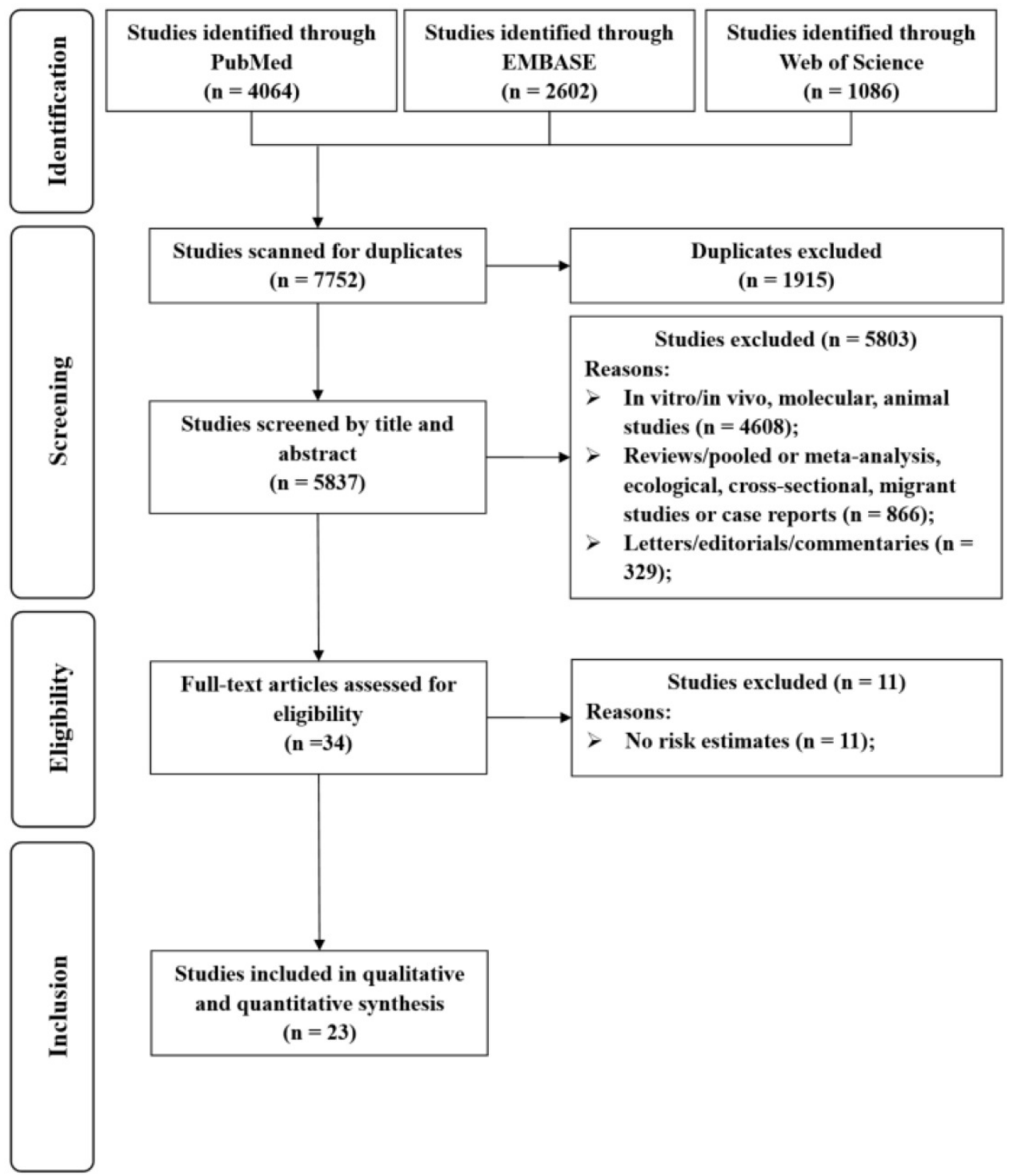

Figure 1. Selection of studies for inclusion in this meta-analysis. 


\section{Results}

\section{Search results, study characteristics, and quality assessment}

The detailed processes of literature screening, study selection, and study exclusion are summarized in Figure 1. The initial search retrieved 7752 unique reports. After removing duplicates and screening the titles and abstracts, the reviewers judged that 34 articles were potentially eligible for inclusion and thus consequently subjected to full-text review. After exclusion, 23 studies were included in the meta-analysis.

Table 1 presents the main characteristics of the 23 included studies. These studies were published from
1991 to 2018 and included a total of 10,612 LC patients with a range of 99-2351 cases among the individual studies. These 23 reports were designed as retrospective $(n=16)$ and prospective studies $(n=7)$. The majority of the included studies were conducted in North America $(n=9)$, seven in Asia, and six in Europe. More than half of the included studies adjusted for age at diagnosis/surgery $(\mathrm{n}=16)$ and TNM stage $(\mathrm{n}=14)$, while less than half adjusted for performance status $(n=11)$, sex $(n=10)$, histology ( $n$ $=10)$, and surgery $(n=8)$. Fewer studies adjusted for systemic inflammatory response markers $(n=6)$, blood transfusion $(n=5)$, body mass index $(n=2)$, and chemotherapy $(n=3)$.

Table 1. Characteristics of 23 studies included in the meta-analysis

\begin{tabular}{|c|c|c|c|c|c|c|c|}
\hline $\begin{array}{l}\text { First author, [ref], year, } \\
\text { country }\end{array}$ & Study design & $\begin{array}{l}\text { No. of } \\
\text { cases }\end{array}$ & $\begin{array}{l}\text { TNM } \\
\text { stage }\end{array}$ & Histology & $\begin{array}{l}\text { Anemia cut-off } \\
\text { (unit) }\end{array}$ & Outcome & Adjustment \\
\hline Zhang et al. [8], 2018, China & Retrospective cohort & 416 & I-IV & NSCLC & $\begin{array}{l}<120(\mathrm{M}) \leq 110 \\
(\mathrm{~F})(\mathrm{g} / \mathrm{L})\end{array}$ & OS & $\begin{array}{l}\text { Age, sex, TNM stage, PS, Lung lobectomy, chemotherapy, } \\
\text { and radiotherapy }\end{array}$ \\
\hline $\begin{array}{l}\text { Holgersson et al. [34], 2017, } \\
\text { Multi-centers }\end{array}$ & $\mathrm{RCT}$ & 99 & IIIB-IV & NSCLC & $<110(\mathrm{~g} / \mathrm{L})$ & OS & $\begin{array}{l}\text { Age at diagnosis, sex, histology, PS, white blood cell and } \\
\text { platelet at baseline and treatment arm }\end{array}$ \\
\hline $\begin{array}{l}\text { Holgersson et al. [35], 2017, } \\
\text { Sweden }\end{array}$ & $\mathrm{RCT}$ & 222 & $\begin{array}{l}\text { IIIA- } \\
\text { IIIB }\end{array}$ & NSCLC & $<110(\mathrm{~g} / \mathrm{L})$ & OS & $\begin{array}{l}\text { Age at diagnosis, sex, histology, PS, white blood cell and } \\
\text { platelet, weight loss, and TNM stage }\end{array}$ \\
\hline Lee et al. [12], 2017, Koera & Retrospective cohort & 135 & IIIB-IV & NSCLC & $\begin{array}{l}<13(\mathrm{M})<12(\mathrm{~F}) \\
(\mathrm{g} / \mathrm{dL})\end{array}$ & OS & N/A \\
\hline Liu et al. [13], 2017, China & Retrospective cohort & 139 & $\mathrm{ED} / \mathrm{LD}$ & SCLC & $\mathrm{N} / \mathrm{A}$ & OS & N/A \\
\hline Cata et al. [14], 2016, USA & Retrospective cohort & 861 & I & NSCLC & $\begin{array}{l}<13(\mathrm{M})<12(\mathrm{~F}) \\
(\mathrm{g} / \mathrm{dL})\end{array}$ & RFS/OS & $\begin{array}{l}\text { Age, body mass index, sex, PS, histology, preoperative } \\
\text { neutrophil-to-lymphocyte ratio, blood transfusion, and } \\
\text { surgery type }\end{array}$ \\
\hline Ng et al. [36], 2012, USA & Prospective cohort & 361 & IA-IB & NSCLC & $\begin{array}{l}<13(\mathrm{M})<12(\mathrm{~F}) \\
(\mathrm{g} / \mathrm{dL})\end{array}$ & DFS/OS & Age, sex, blood transfusion, and TNM stage \\
\hline Wu et al. [16], 2012, China & Retrospective cohort & 200 & ED & SCLC & $\mathrm{N} / \mathrm{A}$ & OS & $\mathrm{N} / \mathrm{A}$ \\
\hline Park et al. [17], 2009, Koera & Retrospective cohort & 316 & IIIB-IV & NSCLC & $<11(\mathrm{~g} / \mathrm{dL})$ & OS & N/A \\
\hline $\begin{array}{l}\text { Chamogeorgakis et al. [18], } \\
\text { 2008, USA }\end{array}$ & Retrospective cohort & 214 & IA-IIB & NSCLC & $\leq 12(\mathrm{~g} / \mathrm{dL})$ & CSM/OS & N/A \\
\hline $\begin{array}{l}\text { Panagopoulos et al. [20], } \\
\text { 2008, Greece }\end{array}$ & Retrospective cohort & 331 & I-IV & NSCLC & $<12(\mathrm{~g} / \mathrm{dL})$ & OS & $\begin{array}{l}\text { Age, sex, operation severity, operation type, histology, } \\
\text { TNM stage postoperative hospital stay, blood transfusion } \\
\text { and number of red blood cell units transfused }\end{array}$ \\
\hline $\begin{array}{l}\text { Tomita et al. [21], 2008, } \\
\text { Japan }\end{array}$ & Retrospective cohort & 240 & I-IV & NSCLC & $\mathrm{N} / \mathrm{A}$ & OS & $\begin{array}{l}\text { Age, sex, histology, TNM stage, and preoperative serum } \\
\text { carcinoma embryonic antigen level }\end{array}$ \\
\hline $\begin{array}{l}\text { Ademuyiwa et al. [22], } \\
\text { 2007, USA }\end{array}$ & RCT & 203 & $\begin{array}{l}\text { IIIA- } \\
\text { IIIB }\end{array}$ & NSCLC & $<12(\mathrm{~g} / \mathrm{dL})$ & OS & $\begin{array}{l}\text { Age, sex, ethnicity, body mass index, PS, FEV } 1 \text {, smoking, } \\
\text { use of positron emission tomography scan in staging and } \\
\text { stage }\end{array}$ \\
\hline $\begin{array}{l}\text { Gauthier et al. [37], 2007, } \\
\text { Canada }\end{array}$ & $\mathrm{RCT}$ & 482 & IB- II & NSCLC & $<120(\mathrm{~g} / \mathrm{L})$ & OS & $\begin{array}{l}\text { Age, sex, PS, TNM stage, histology, type of surgery, and } \\
\text { baseline lactic dehydrogenase }\end{array}$ \\
\hline $\begin{array}{l}\text { Mandrekar et al. [23], 2006, } \\
\text { USA and Canada }\end{array}$ & $\mathrm{RCT}$ & 1053 & IIIB-IV & NSCLC & $\begin{array}{l}<13.2(\mathrm{M})<11.5 \\
(\mathrm{~F})(\mathrm{g} / \mathrm{dL})\end{array}$ & OS & $\begin{array}{l}\text { Age, sex, PS, TNM stage, BMI, white blood cell, and } \\
\text { platelet count }\end{array}$ \\
\hline Aoe et al. [24], 2005, Japan & Retrospective cohort & 611 & I-IV & LC & $\begin{array}{l}<13(\mathrm{M})<12(\mathrm{~F}) \\
(\mathrm{g} / \mathrm{dL})\end{array}$ & OS & $\begin{array}{l}\text { Age, sex, PS, histology, TNM stage, and lactic } \\
\text { dehydrogenase }\end{array}$ \\
\hline $\begin{array}{l}\text { Berardi et al. [25], 2005, } \\
\text { Italy }\end{array}$ & Retrospective cohort & 439 & IA-IIIB & NSCLC & $\leq 10(\mathrm{~g} / \mathrm{dL})$ & OS & $\begin{array}{l}\text { Age, sex, smoking, PS, histology, TNM stage, type of } \\
\text { surgery, and transfusions }\end{array}$ \\
\hline $\begin{array}{l}\text { Yovino et al. [26], 2005, } \\
\text { USA }\end{array}$ & Retrospective cohort & 125 & I-II & NSCLC & $<12(\mathrm{~g} / \mathrm{dL})$ & OS & $\begin{array}{l}\text { Age, sex, histology, TNM stage, p53 status, and type of } \\
\text { surgery }\end{array}$ \\
\hline $\begin{array}{l}\text { Rzyman et al. [27], 2003, } \\
\text { Poland }\end{array}$ & Retrospective cohort & 493 & I-IV & NSCLC & $\leq 12(\mathrm{~g} / \mathrm{dL})$ & OS & $\begin{array}{l}\text { Age, sex, amount and type of transfused blood, } \\
\text { sedimentation rate, histology, tumor location, type of } \\
\text { resection, and TNM stage }\end{array}$ \\
\hline Jazieh et al. [28], 2000, USA & Retrospective cohort & 454 & I-II & NSCLC & $\leq 10(\mathrm{~g} / \mathrm{dL})$ & EFS/OS & Age, sex, race, TNM stage, histology, and type of surgery \\
\hline $\begin{array}{l}\text { Wigren et al. [31], 1997, } \\
\text { Finland }\end{array}$ & Retrospective cohort & 502 & I-IV & NSCLC & $\leq 125(\mathrm{~g} / \mathrm{L})$ & OS & TNM stage, symptoms, PS, and tumor size \\
\hline $\begin{array}{l}\text { Takigawa et al. [32], 1996, } \\
\text { Japan }\end{array}$ & Retrospective cohort & 185 & III-IV & NSCLC & $\leq 11(\mathrm{~g} / \mathrm{dL})$ & OS & PS, TNM stage, calcium \\
\hline Albain et al. [33], 1991, USA & RCT & 2351 & I-IV & NSCLC & $\leq 11(\mathrm{~g} / \mathrm{dL})$ & OS & $\begin{array}{l}\text { Lactic dehydrogenase, calcium metastasis, therapy, year } \\
\text { of registration, other therapy groupings, sex, age, } \\
\text { smoking, weight loss, and alkaline phosphatase }\end{array}$ \\
\hline
\end{tabular}

CSM, cancer-specific mortality; DFS, disease-free survival; ED, extensive stage; EFS, event-free survival; F, female; LC, lung cancer; LD, limited stage; M, male; NSCLC, non-small cell lung cancer; N/A, not available; OS, overall survival; PFS, progression-free survival; PS, performance status; RCT, randomized controlled trial; RFS, recurrence-free survival; SCLC, small cell lung cancer. 
Table 2. Methodological quality of all included cohort studies

\begin{tabular}{|c|c|c|c|c|c|c|c|c|}
\hline First author (reference), year & $\begin{array}{l}\text { Representativeness } \\
\text { of the exposed cohort }\end{array}$ & $\begin{array}{l}\text { Selection of } \\
\text { the } \\
\text { unexposed } \\
\text { cohort }\end{array}$ & $\begin{array}{l}\text { Ascertainment } \\
\text { of exposure }\end{array}$ & $\begin{array}{l}\text { Outcome of } \\
\text { interest not } \\
\text { present at start of } \\
\text { study }\end{array}$ & $\begin{array}{l}\text { Control for } \\
\text { important factor or } \\
\text { additional factor }\end{array}$ & $\begin{array}{l}\text { Assessment of } \\
\text { outcome }\end{array}$ & $\begin{array}{l}\text { Follow-up long } \\
\text { enough for } \\
\text { outcomes to } \\
\text { occur } \ddagger\end{array}$ & $\begin{array}{l}\text { Adequacy } \\
\text { of cohort } \\
\text { follow-up } \S\end{array}$ \\
\hline Zhang et al. [8], 2018 & * & * & * & * & ** & * & * & $*$ \\
\hline Holgersson et al.[34], 2017 & * & * & * & * & ** & * & _ & * \\
\hline Holgersson et al. [35], 2017 & * & * & * & * & ** & * & * & * \\
\hline Lee et al. [12], 2017 & * & * & * & $*$ & - & * & - & $*$ \\
\hline Liu et al. [13], 2017 & * & * & * & * & _ & * & * & * \\
\hline Cata et al. [14], 2016 & * & * & * & * & $* *$ & * & * & * \\
\hline $\mathrm{Ng}$ et al. [36], 2012 & * & * & * & * & ** & * & * & * \\
\hline Wu et al. [16], 2012 & * & * & * & * & _- & * & * & * \\
\hline Park et al. [17], 2009 & * & * & * & * & - & * & * & * \\
\hline $\begin{array}{l}\text { Chamogeorgakis et al. [18], } \\
2008\end{array}$ & * & * & * & * & - & * & * & $*$ \\
\hline Panagopoulos et al. [20], 2008 & * & * & * & * & $* *$ & * & * & * \\
\hline Tomita et al. [21], 2008 & * & * & * & * & * & * & * & * \\
\hline Ademuyiwa et al. [22], 2007 & * & * & * & * & * & * & * & * \\
\hline Gauthier et al. [37], 2007 & * & * & * & * & * & * & - & * \\
\hline Mandrekar et al. [23], 2006 & * & * & * & * & - & * & * & * \\
\hline Aoe et al. [24], 2005 & * & * & * & $*$ & ** & * & - & $*$ \\
\hline Berardi et al. [25], 2005 & * & * & * & * & ** & * & * & * \\
\hline Yovino et al. [26], 2005 & * & * & * & * & $* *$ & * & * & * \\
\hline Rzyman et al. [27], 2003 & * & * & * & * & ** & * & * & * \\
\hline Jazieh et al. [28], 2000 & * & * & * & * & $* *$ & * & * & * \\
\hline Wigren et al. [31], 1997 & * & * & * & * & $* *$ & * & - & * \\
\hline Takigawa et al. [32], 1996 & * & * & * & * & $* *$ & * & * & * \\
\hline Albain et al. [33], 1991 & * & * & * & * & * & * & _ & * \\
\hline
\end{tabular}

A study could be awarded a maximum of one star for each item except for the item "Control for important factor or additional factor."

† A maximum of two stars could be awarded for this item. Studies that controlled for age at diagnosis/TNM stage received one star, whereas those that controlled for other important confounders (i.e., performance status, blood transfusion, and surgery/chemotherapy) received an additional star.

$\ddagger$ A cohort study with a median follow-up time $>1$ year was assigned one star.

$\S$ A cohort study with a follow-up rate $>75 \%$ was assigned one star.

The quality assessment characteristics of the included studies are shown in Table 2. The major difference among the included studies was the control for an important factor or an additional factor category; 13 of the included studies were assigned two full scores. Six studies [12, 24, 31, 33, 34, 37] had follow-up periods of less than one year or were not mentioned; therefore, these studies were not assigned a score when testing for whether the follow-up duration was sufficiently long for outcomes to occur.

\section{Pre-operative anemia and OS of LC patients}

As compared with LC patients without anemia, those with pre-operative anemia were at approximately a 1.58-fold greater risk of death (summarized HR $=1.58 ; 95 \% \mathrm{CI}=1.44-1.75$ ) (Figure $2)$, with moderate heterogeneity $\left(I^{2}=53.1 \%\right)$. Funnel plot and statistical analyses showed no evidence of publication bias (Figure 3).

\section{Subgroup and sensitivity analyses}

To determine whether the study characteristics and adjustment for potential confounders had any impact on risk estimates, numerous subgroup analyses stratified by these issues were performed. Significant positive findings were observed throughout these subgroup analyses (Table 3). Sensitivity analyses using an alternative statistical model (fixed-effects $\mathrm{HR}=1.50,95 \% \mathrm{CI}=1.42-1.60$ ) and by excluding five studies [12, 13, 16-18] without adjustment for any potential confounders (summarized $\mathrm{HR}=1.54,95 \% \mathrm{CI}=1.38-1.72 ; I^{2}=$ $56.4 \%$ ) showed robust findings. Additionally, the sensitivity analysis showed that the summarized HR 
ranged from $1.55\left(95 \% \mathrm{CI}=1.41-1.69 ; I^{2}=44.3 \%\right.$; exclusion of Takigawa et al. [32]) to 1.62 (95\% CI = $1.48-1.77 ; I^{2}=37.0 \%$; exclusion of Ademuyiwa et al. [22]) (Figure 4). When the studies that contributed the largest amount to heterogeneity until $I^{2}$ was less than $50 \%$ were sequentially excluded, the summarized HR was $1.62\left(95 \% \mathrm{CI}=1.48-1.77 ; I^{2}=37.0 \%\right)$, which was similar to the original estimate.

\section{Discussion}

This systematic review and meta-analysis with a large sample size (comprising 10,612 LC patients) provides support for the hypothesis that pre-operative anemia is associated with increased mortality among LC patients. This finding should improve the attention of both patents and clinicians to better management of pre-operative anemia before further treatment of LC.

Table 3. Risk estimate summary of the relationship between pre-operative anemia and OS of LC patients

\begin{tabular}{|c|c|c|c|c|c|c|}
\hline & No. of studies & HR & $95 \% \mathrm{CI}$ & $I^{2}(\%)$ & $P_{\mathrm{h}}^{\dagger}$ & $P_{\mathrm{h}^{\ddagger}}$ \\
\hline Overall & 23 & 1.58 & $1.44-1.75$ & 53.1 & 0.001 & \\
\hline \multicolumn{7}{|l|}{ Subgroup analyses } \\
\hline Study design & & & & & & 0.104 \\
\hline Retrospective & 16 & 1.67 & $1.49-1.86$ & 45.2 & 0.026 & \\
\hline Prospective & 7 & 1.40 & $1.19-1.64$ & 46.2 & 0.084 & \\
\hline Geographic location & & & & & & 0.116 \\
\hline Asia & 8 & 1.73 & $1.48-2.02$ & 54.8 & 0.030 & \\
\hline North America & 9 & 1.61 & $1.34-1.94$ & 65.3 & 0.003 & \\
\hline Europe & 6 & 1.41 & $1.22-1.62$ & 0 & 0.815 & \\
\hline Number of cases (median) & & & & & & 0.818 \\
\hline$\geq 300$ & 13 & 1.59 & $1.44-1.76$ & 34.3 & 0.108 & \\
\hline$<300$ & 10 & 1.61 & $1.31-1.97$ & 64.3 & 0.003 & \\
\hline TNM stage & & & & & & 0.415 \\
\hline All & 8 & 1.50 & $1.37-1.65$ & 0 & 0.546 & \\
\hline Advanced stage & 9 & 1.63 & $1.34-1.99$ & 73.3 & $<0.001$ & \\
\hline Early stage & 6 & 1.74 & $1.33-2.28$ & 47.8 & 0.088 & \\
\hline Histology & & & & & & 0.823 \\
\hline Non-small cell & 20 & 1.59 & $1.42-1.77$ & 57.3 & 0.001 & \\
\hline Small cell & 2 & 1.91 & $1.37-2.66$ & 0 & 0.941 & \\
\hline Quality of study & & & & & & 0.847 \\
\hline Low risk & 20 & 1.59 & $1.42-1.76$ & 56.9 & 0.001 & \\
\hline High risk & 3 & 1.63 & $1.28-2.08$ & 13.3 & 0.316 & \\
\hline \multicolumn{7}{|c|}{ Adjustment for potential confounders or risk factors } \\
\hline Age at diagnosis/surgery & & & & & & 0.125 \\
\hline Yes & 16 & 1.51 & $1.35-1.67$ & 47.7 & 0.018 & \\
\hline No & 7 & 1.80 & $1.49-2.17$ & 48.6 & 0.069 & \\
\hline Sex & & & & & & 0.303 \\
\hline Yes & 14 & 1.65 & $1.48-1.85$ & 30.0 & 0.144 & \\
\hline No & 9 & 1.51 & $1.30-1.76$ & 61.6 & 0.005 & \\
\hline Body mass index & & & & & & 0.119 \\
\hline Yes & 2 & 1.33 & $1.06-1.69$ & 78.8 & 0.030 & \\
\hline No & 21 & 1.64 & $1.48-1.81$ & 39.3 & 0.034 & \\
\hline TNM stage & & & & & & 0.892 \\
\hline Yes & 14 & 1.58 & $1.40-1.77$ & 49.1 & 0.020 & \\
\hline No & 9 & 1.62 & $1.35-1.94$ & 61.3 & 0.008 & \\
\hline Histology & & & & & & 0.375 \\
\hline Yes & 14 & 1.49 & $1.30-1.70$ & 28.9 & 0.179 & \\
\hline No & 9 & 1.66 & $1.45-1.91$ & 64.6 & 0.001 & \\
\hline Performance status & & & & & & 0.102 \\
\hline Yes & 11 & 1.47 & $1.29-1.67$ & 58.9 & 0.007 & \\
\hline No & 12 & 1.73 & $1.53-1.96$ & 22.8 & 0.219 & \\
\hline Surgery & & & & & & 0.232 \\
\hline Yes & 8 & 1.74 & $1.46-2.07$ & 40.3 & 0.110 & \\
\hline No & 15 & 1.52 & $1.36-1.70$ & 53.4 & 0.008 & \\
\hline Blood transfusion & & & & & & 0.374 \\
\hline Yes & 5 & 1.45 & $1.24-1.70$ & 0 & 0.978 & \\
\hline No & 18 & 1.64 & $1.46-1.85$ & 63.3 & $<0.001$ & \\
\hline SIR markers & & & & & & 0.208 \\
\hline Yes & 6 & 1.46 & $1.29-1.65$ & 0 & 0.817 & \\
\hline No & 17 & 1.67 & $1.47-1.89$ & 64.0 & $<0.001$ & \\
\hline
\end{tabular}




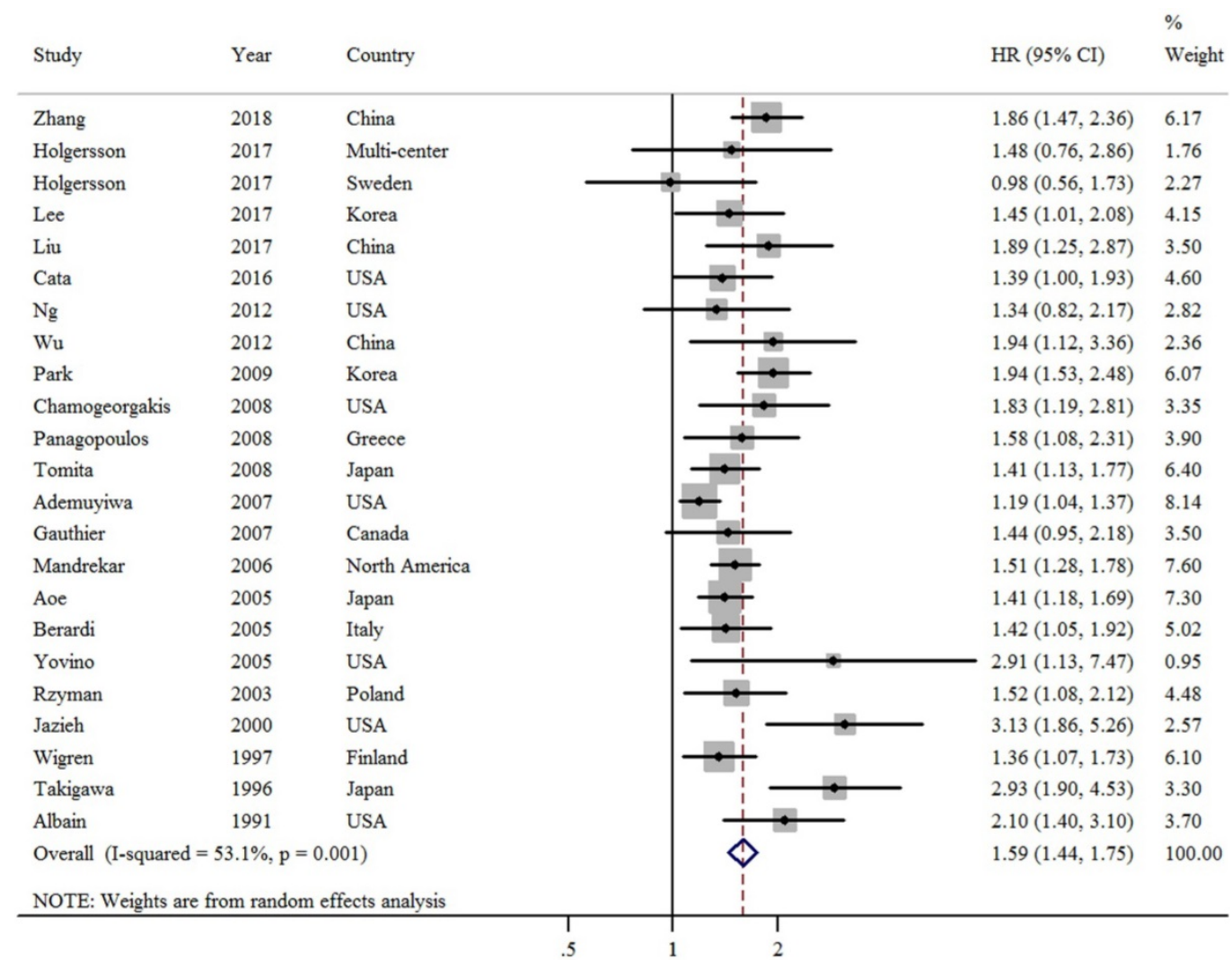

Hazard Ratio

Figure 2. Forest plot (random effects model) of pre-operative anemia and OS of LC patients. The squares indicate study-specific hazard ratios (size of the square reflects the study-specific statistical weight); the horizontal lines indicate $95 \% \mathrm{Cls}$; and the diamond indicates the summary hazard ratio estimate with its $95 \% \mathrm{Cl}$.



Figure 3. Test for publication bias for OS through Begg's funnel plot. HR, hazard ratio; SE, standard error. The circles alone are real studies. The vertical lines represent the summary effect estimates and the dashed lines represent pseudo- $95 \% \mathrm{Cl}$ limits. 


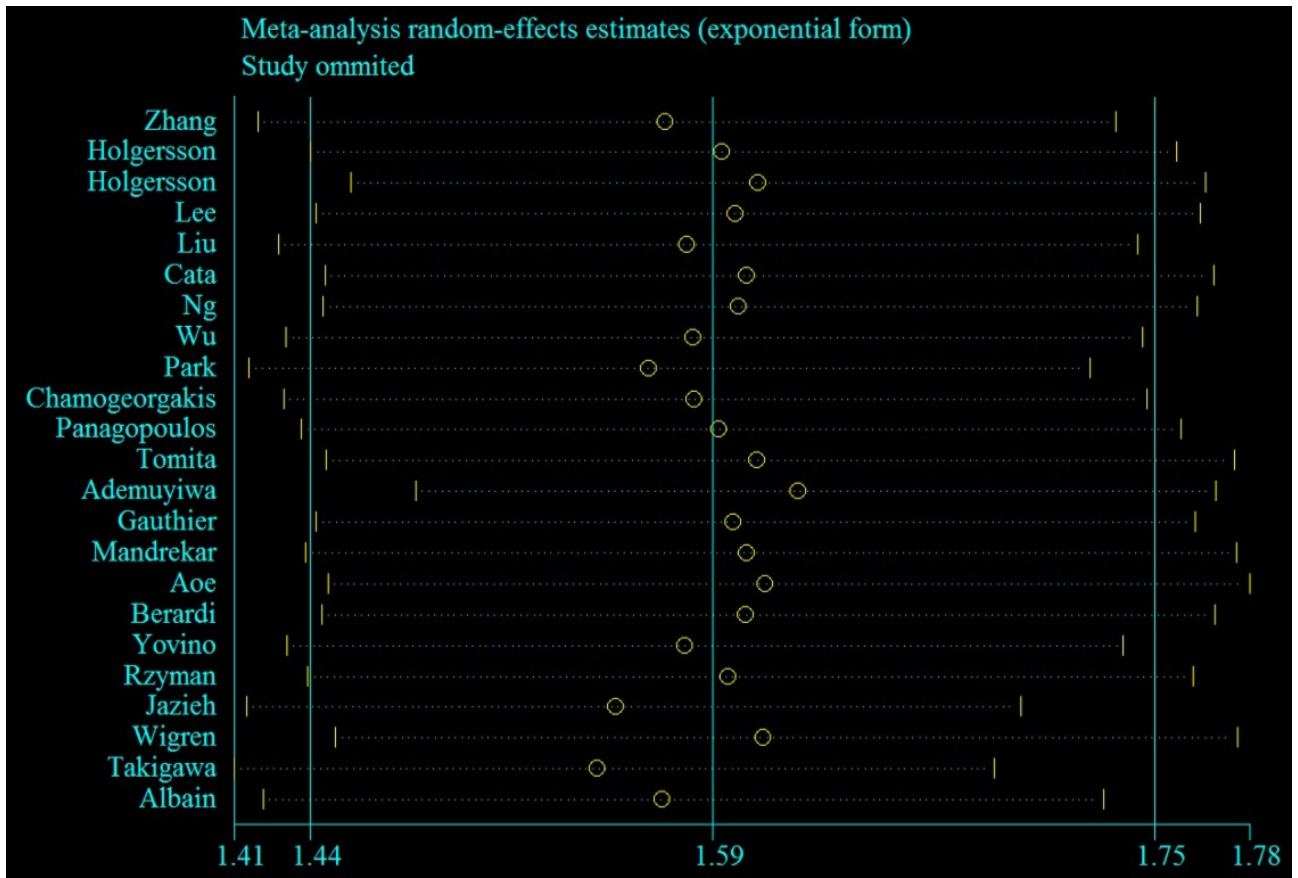

Figure 4. Sensitivity plot corresponding to the relationship between pre-operative anemia and OS of LC patients. The circle indicates the study-specific hazard ratio after excluding the present study; the horizontal dotted lines indicate $95 \% \mathrm{Cls}$.

One previous systematic review reported an overall estimate of the effect of anemia on survival in patients with malignant disease [11]. In 2001, Caro et al. [11] found that the relative risk of death was increased by $19 \%$ among anemic patients with lung carcinoma. However, this report did not provide details of the extracted information. Furthermore, several included studies [47-51] failed to a risk estimate in the primary analysis. In comparison, the results of the present study, which were primarily based on the most comprehensive published studies, suggested a relatively stronger relationship between pre-operative anemia and survival of LC patients. Of note, these findings were robust in subgroup analyses, which were not performed by Caro et al. [11] in 2001.

The precise mechanisms underlying the association between pre-operative anemia and survival in LC patients have not been fully elucidated. In general, cancer-related anemia is due to multiple etiologies, including blood loss, functional iron deficiency, erythropoietin deficiency from renal disease, and bone marrow involvement with cancer, as well as other factors [52]. One hypothesis is that tumor hypoxia may stimulate angiogenesis, which is a marker of increased tumor aggressiveness [53]. Tumor cells are known to secrete various soluble molecules, including interleukin- 6 and tumor necrosis factor- $\alpha$ [8]. These molecules can decrease $\mathrm{Hb}$ concentrations by changing the hematopoietic environment $[54,55]$, suppressing erythropoiesis and erythropoietin [56], and impairing the erythropoietin response of erythroid progenitor cells [57]. Additionally, in patients with bone metastasis, bone marrow involvement may lead to bone morrow failure, which may then cause low $\mathrm{Hb}$ levels [58] and subsequently lead to hypoxia, which could induce genomic changes and enhance the development of malignancy [59]. Hypoxia may also boost tumor angiogenesis and accelerate metastasis [60]. Furthermore, hypoxia may enhance tumor cell resistance to chemotherapy and radiotherapy through the development of multi-drug resistance [61].

The strengths of this study include the systematic and rigorous approach to the identification of epidemiological studies investigating the impact of pre-operative anemia on survival of LC patients. Furthermore, to the best of our knowledge, the present study included the most published studies and had the largest sample size, which allowed for numerous preplanned subgroup analyses to explore differences in the risks for mortality.

This study also had some limitations that should be addressed. First, the majority of the included studies were retrospective, which might have had potential selection and recall biases, thereby diminishing the general credibility of these findings. However, no significant difference was found in the subgroup analysis stratified by study design. Furthermore, most of included studies were scored as low risk after quality assessment. The only three studies with high risk might be attributed to the 
adjustment for limited confounders as well as short follow-up periods. Second, the cut-off value and unit of pre-operative anemia varied. For example, six of the included studies defined pre-operative anemia according to patient sex. However, three studies only reported pre-operative anemia as low or normal. Furthermore, five studies used $\mathrm{g} / \mathrm{L}$ as the unit of anemia. In contrast, 15 studies used $\mathrm{g} / \mathrm{dL}$ as the unit of anemia. Third, since the majority of the included studies were observational, the association between pre-operative anemia and prognosis of LC may have resulted from unmeasured or residual confounding by other factors. Pre-operative anemia in LC patients may be associated with age at diagnosis, body mass index, TNM stage, histology, performance status, and blood transfusion, which possibly could confound the aforementioned associations. For example, several previous studies have mentioned that blood transfusion might affect adversely the survival of LC patients, especially for stage I non-small cell LC patients [20]. Although the findings were robust in studies that adjusted for these potential confounders, not all were fully adjusted. Notably, five studies provided the risk estimates on the basis of a univariate model, but without adjustment for any confounders. Interestingly, the results of the sensitivity analysis were robust when excluding these five studies. Furthermore, the results of meta-regression analyses found no evidence that these findings differed significantly between studies adjusted for these confounders or not. Fourth, since a limited number of studies reported secondary outcomes (i.e., progression-free survival, recurrence-free survival, or event-free survival), the main focus of the present study was the association between pre-operative anemia and OS of LC patients. Further studies are warranted to provide more information on this issue.

The findings of the present study not only indicate a significant association between pre-operative anemia and an increased risk of LC mortality, but also emphasize the role of systematic reviews to examine focused clinical questions. Pre-operative anemia might be a useful indicator of the prognosis of LC patients. Clinicians should fully consider the status and severity of pre-operative anemia when deciding on an appropriate individualized LC management regime.

\section{Author Contributions}

Study concepts: X-XF. Study design: YL, Y-PB, and X-XF. Data acquisition: YL, Y-PB, and X-XF. Quality control of data and algorithms: Z-FZ and C-RJ. Data analysis and interpretation: $\mathrm{YL}$ and $\mathrm{X}-\mathrm{XF}$. Statistical analysis: YL and X-XF. Manuscript preparation: YL, Y-PB, Z-FZ, C-RJ, ZX, and X-XF. Manuscript editing: $X-X F$. Manuscript review: $Y L$, Y-PB, Z-FZ, C-RJ, ZX, and X-XF.

\section{Competing Interests}

The authors have declared that no competing interest exists.

\section{References}

1. Torre LA, Bray F, Siegel RL, et al. Global cancer statistics, 2012. CA Cancer J Clin. 2015; 65: 87-108.

2. Morgensztern D, Ng SH, Gao F, et al. Trends in stage distribution for patients with non-small cell lung cancer: a National Cancer Database survey. J Thorac Oncol. 2010; 5: 29-33.

3. Ettinger DS, Kuettel M, Malin J, et al. NCCN Roundtable: What Are the Characteristics of an Optimal Clinical Practice Guideline? J Natl Compr Canc Netw. 2015; 13: 640-642.

4. Alberg AJ, Brock MV, Ford JG, et al. Epidemiology of lung cancer: Diagnosis and management of lung cancer, 3rd ed: American College of Chest Physicians evidence-based clinical practice guidelines. Chest. 2013; 143: e1S-e29S.

5. Palma JF, Das P, Liesenfeld O. Lung cancer screening: utility of molecular applications in conjunction with low-dose computed tomography guidelines. Expert Rev Mol Diagn. 2016; 16: 435-447.

6. Bearz A, Berretta M, Tirelli U. Clinical Effectiveness and Cost-effectiveness of Target Therapies for Adult Patients with Locally Advanced or Metastatic Non-small Cell Lung Cancer: A Systematic Review. Curr Cancer Drug Targets. 2018; 18: 405-409.

7. Greenhalgh J, Dwan K, Boland A, et al. First-line treatment of advanced epidermal growth factor receptor (EGFR) mutation positive non-squamous non-small cell lung cancer. Cochrane Database Syst Rev. 2016: CD010383.

8. Zhang $\mathrm{YH}, \mathrm{Lu} \mathrm{Y}, \mathrm{Lu} \mathrm{H}$, et al. Pre-treatment hemoglobin levels are an independent prognostic factor in patients with non-small cell lung cancer. Mol Clin Oncol. 2018; 9: 44-49.

9. Mercadante S, Gebbia V, Marrazzo A, et al. Anaemia in cancer: pathophysiology and treatment. Cancer Treat Rev. 2000; 26: 303-311.

10. Birgegard G, Aapro MS, Bokemeyer C, et al. Cancer-related anemia: pathogenesis, prevalence and treatment. Oncology. 2005; 68 Suppl 1: 3-11.

11. Caro JJ, Salas M, Ward A, et al. Anemia as an independent prognostic factor for survival in patients with cancer: a systemic, quantitative review. Cancer. 2001; 91: 2214-2221.

12. Lee S, Eo W, Jeon H, et al. Prognostic Significance of Host-related Biomarkers for Survival in Patients with Advanced Non-Small Cell Lung Cancer. J Cancer. 2017; 8: 2974-2983.

13. Liu D, Huang Y, Li L, et al. High neutrophil-to-lymphocyte ratios confer poor prognoses in patients with small cell lung cancer. BMC Cancer. 2017; 17: 882.

14. Cata JP, Gutierrez C, Mehran RJ, et al. Preoperative anemia, blood transfusion, and neutrophil-to-lymphocyte ratio in patients with stage i non-small cell lung cancer. Cancer Cell Microenviron. 2016; 3: e1116.

15. Tanriverdi O, Cokmert S, Oktay E, et al. Prognostic significance of the baseline serum uric acid level in non-small cell lung cancer patients treated with first-line chemotherapy: a study of the Turkish Descriptive Oncological Researches Group. Med Oncol. 2014; 31: 217.

16. Wu C, Li F, Jiao SC. Prognostic factors for survival of patients with extensive stage small cell lung cancer--a retrospective single institution analysis. Asian Pac J Cancer Prev. 2012; 13: 4959-4962.

17. Park MJ, Lee J, Hong JY, et al. Prognostic model to predict outcomes in nonsmall cell lung cancer patients treated with gefitinib as a salvage treatment. Cancer. 2009; 115: 1518-1530.

18. Chamogeorgakis T, Anagnostopoulos C, Kostopanagiotou G, et al. Does anemia affect outcome after lobectomy or pneumonectomy in early stage lung cancer patients who have not received neo-adjuvant treatment? Thorac Cardiovasc Surg. 2008; 56: 148-153.

19. Garcia PJ, Gonzalez BF, Moldes RM, et al. [Impact of hemoglobin level on lung cancer survival]. Med Clin (Barc). 2008; 131: 601-604.

20. Panagopoulos ND, Karakantza M, Koletsis E, et al. Influence of blood transfusions and preoperative anemia on long-term survival in patients operated for non-small cell lung cancer. Lung Cancer. 2008; 62: 273-280.

21. Tomita M, Shimizu T, Hara M, et al. Impact of preoperative hemoglobin level on survival of non-small cell lung cancer patients. Anticancer Res. 2008; 28: 1947-1950

22. Ademuyiwa FO, Johnson CS, White AS, et al. Prognostic factors in stage III non-small-cell lung cancer. Clin Lung Cancer. 2007; 8: 478-482.

23. Mandrekar SJ, Schild SE, Hillman SL, et al. A prognostic model for advanced stage nonsmall cell lung cancer. Pooled analysis of North Central Cancer Treatment Group trials. Cancer. 2006; 107: 781-792.

24. Aoe K, Hiraki A, Maeda T, et al. Serum hemoglobin level determined at the first presentation is a poor prognostic indicator in patients with lung cancer. Intern Med. 2005; 44: 800-804 
25. Berardi R, Brunelli A, Tamburrano T, et al. Perioperative anemia and blood transfusions as prognostic factors in patients undergoing resection for non-small cell lung cancers. Lung Cancer. 2005; 49: 371-376.

26. Yovino S, Kwok Y, Krasna M, et al. An association between preoperative anemia and decreased survival in early-stage non-small-cell lung cancer patients treated with surgery alone. Int J Radiat Oncol Biol Phys. 2005; 62: 1438-1443.

27. Rzyman W, Dziadziuszko R, Skokowski J, et al. The influence of blood transfusion on survival in operated non-small cell lung cancer patients. J Thorac Cardiovasc Surg. 2003; 126: 755-760.

28. Jazieh AR, Hussain M, Howington JA, et al. Prognostic factors in patients with surgically resected stages I and II non-small cell lung cancer. Ann Thorac Surg. 2000; 70: 1168-1171.

29. Ylisirnio S, Sassi ML, Risteli J, et al. Serum type I collagen degradation markers, ICTP and CrossLaps, are factors for poor survival in lung cancer. Anticancer Res. 1999; 19: 5577-5581.

30. Watine J, Charet JC. Do blood cell counts have an independent prognostic value in primary lung cancer? Hematol Cell Ther. 1998; 40: 99-106.

31. Wigren $\mathrm{T}$, Oksanen $\mathrm{H}$, Kellokumpu-Lehtinen P. A practical prognostic index for inoperable non-small-cell lung cancer. J Cancer Res Clin Oncol. 1997; 123: $259-266$

32. Takigawa N, Segawa Y, Okahara M, et al. Prognostic factors for patients with advanced non-small cell lung cancer: univariate and multivariate analyses including recursive partitioning and amalgamation. Lung Cancer. 1996; 15: 67-77.

33. Albain KS, Crowley JJ, LeBlanc $M$, et al. Survival determinants in extensive-stage non-small-cell lung cancer: the Southwest Oncology Group experience. J Clin Oncol. 1991; 9: 1618-1626.

34. Holgersson G, Bergqvist M, Nilsson J, et al. The Prognostic Value of Pre-Treatment Leukocytosis in Patients with Previously Treated, Stage IIIB/IV Non-Small Cell Lung Cancer Treated with the IGF-1R Pathway Modulator AXL1717 or Docetaxel; a Retrospective Analysis of a Phase II Trial. Asian Pac J Cancer Prev. 2017; 18: 1555-1560.

35. Holgersson G, Bergstrom S, Hallqvist A, et al. The prognostic value of pre-treatment thrombocytosis in two cohorts of patients with non-small cell lung cancer treated with curatively intended chemoradiotherapy. Neoplasma. 2017; 64: 909-915.

36. $\mathrm{Ng} \mathrm{T}$, Ryder BA, Chern $\mathrm{H}$, et al. Leukocyte-depleted blood transfusion is associated with decreased survival in resected early-stage lung cancer. J Thorac Cardiovasc Surg. 2012; 143: 815-819.

37. Gauthier I, Ding K, Winton T, et al. Impact of hemoglobin levels on outcomes of adjuvant chemotherapy in resected non-small cell lung cancer: the JBR.10 trial experience. Lung Cancer. 2007; 55: 357-363.

38. Stroup DF, Berlin JA, Morton SC, et al. Meta-analysis of observational studies in epidemiology: a proposal for reporting. Meta-analysis Of Observational Studies in Epidemiology (MOOSE) group. JAMA. 2000; 283: 2008-2012

39. Wells GA, Shea B, O'Connell D, et al. The Newcastle-Ottawa Scale (NOS) for assessing the quality of nonrandomised studies in meta-analyses. http://www.ohri.ca/programs/clinical_epidemiology

40. Hamling J, Lee P, Weitkunat R, et al. Facilitating meta-analyses by deriving relative effect and precision estimates for alternative comparisons from a set of estimates presented by exposure level or disease category. Stat Med. 2008; 27: 954-970.

41. Higgins JP, Thompson SG. Quantifying heterogeneity in a meta-analysis. Stat Med. 2002; 21: 1539-58.

42. Begg CB, Mazumdar M. Operating characteristics of a rank correlation test for publication bias. Biometrics. 1994; 50: 1088-1101.

43. Egger M, Davey SG, Schneider M, et al. Bias in meta-analysis detected by a simple, graphical test. BMJ. 1997; 315: 629-634

44. Patsopoulos NA, Evangelou E, Ioannidis JP. Sensitivity of between-study heterogeneity in meta-analysis: proposed metrics and empirical evaluation. Int J Epidemiol. 2008; 37: 1148-1157.

45. Huo YL, Qiao JM, Gao S. Association between antidepressant medication use and epithelial ovarian cancer risk: a systematic review and meta-analysis of observational studies. Br J Clin Pharmacol. 2018; 84: 649-658.

46. Odutayo A, Wong $\mathrm{CX}$, Hsiao AJ, et al. Atrial fibrillation and risks of cardiovascular disease, renal disease, and death: systematic review and meta-analysis. BMJ. 2016; 354: i4482.

47. Yamamoto N, Tamura T, Fukuoka M, et al. Survival and prognostic factors in lung cancer patients treated in phase I trials: Japanese experience. Int J Oncol. 1999; 15: 737-741.

48. Sorensen JB, Badsberg $\mathrm{JH}$, Olsen J. Prognostic factors in inoperable adenocarcinoma of the lung: a multivariate regression analysis of 259 patients. Cancer Res. 1989; 49: 5748-5754

49. Osterlind K, Andersen PK. Prognostic factors in small cell lung cancer: multivariate model based on 778 patients treated with chemotherapy with or without irradiation. Cancer Res. 1986; 46: 4189-4194.

50. Souhami RL, Bradbury I, Geddes DM, et al. Prognostic significance of laboratory parameters measured at diagnosis in small cell carcinoma of the lung. Cancer Res. 1985; 45: 2878-2882.

51. Shinkai T, Eguchi K, Sasaki Y, et al. A prognostic-factor risk index in advanced non-small-cell lung cancer treated with cisplatin-containing combination chemotherapy. Cancer Chemother Pharmacol. 1992; 30: 1-6.

52. Xia L, Guzzo TJ. Preoperative Anemia and Low Hemoglobin Level Are Associated With Worse Clinical Outcomes in Patients With Bladder Cancer
Undergoing Radical Cystectomy: A Meta-Analysis. Clin Genitourin Cancer. 2017; 15: 263-272.e4.

53. Knight K, Wade S, Balducci L. Prevalence and outcomes of anemia in cancer: a systematic review of the literature. Am J Med. 2004; 116 Suppl 7A: 11S-26S.

54. Banzet S, Sanchez H, Chapot R, et al. Interleukin-6 contributes to hepcidin mRNA increase in response to exercise. Cytokine. 2012; 58: 158-161.

55. Sun CC, Vaja V, Babitt JL, et al. Targeting the hepcidin-ferroportin axis to develop new treatment strategies for anemia of chronic disease and anemia of inflammation. Am J Hematol. 2012; 87: 392-400.

56. Wu Y, Antony S, Meitzler JL, et al. Molecular mechanisms underlying chronic inflammation-associated cancers. Cancer Lett. 2014; 345: 164-173.

57. Aapro M, Jelkmann W, Constantinescu SN, et al. Effects of erythropoietin receptors and erythropoiesis-stimulating agents on disease progression in cancer. Br J Cancer. 2012; 106: 1249-1258.

58. Tefferi A, Hudgens S, Mesa R, et al. Use of the Functional Assessment of Cancer Therapy--anemia in persons with myeloproliferative neoplasm-associated myelofibrosis and anemia. Clin Ther. 2014; 36: 560-566.

59. Schwab LP, Peacock DL, Majumdar D, et al. Hypoxia-inducible factor 1alpha promotes primary tumor growth and tumor-initiating cell activity in breast cancer. Breast Cancer Res. 2012; 14: R6.

60. Li S, Meng W, Guan Z, et al. The hypoxia-related signaling pathways of vasculogenic mimicry in tumor treatment. Biomed Pharmacother. 2016; 80: 127-135

61. Milane L, Duan Z, Amiji M. Role of hypoxia and glycolysis in the development of multi-drug resistance in human tumor cells and the establishment of an orthotopic multi-drug resistant tumor model in nude mice using hypoxic pre-conditioning. Cancer Cell Int. 2011; 11: 3 